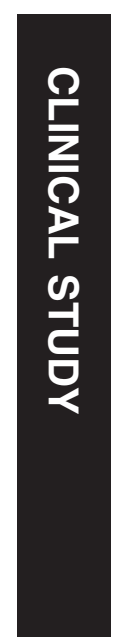

\title{
The relationship between macular cell layer thickness and visual function in different stages of glaucoma
}

${ }^{1}$ Department of Ophthalmology and Visual Sciences, University of Illinois at Chicago, Chicago, IL, USA

${ }^{2}$ Division of Epidemiology and Biostatistics, School of Public Health, University of Illinois at Chicago, Chicago, IL, USA

${ }^{3}$ Department of Ophthalmology, Summa Health System, Akron, Ohio, USA

Correspondence:

TS Vajaranant, Department of Ophthalmology and Visual Sciences, University of Illinois at Chicago, 1855 West Taylor

St, Chicago, IL 60612, USA

Tel: + 1312996 7030;

Fax: + 13124138475

E-mail: thasarat@uic.edu

Received: 17 March 2010 Accepted in revised form: 28 December 2010

Published online: 25 February 2011

This work has previously been presented in part at the Associations for Research in Vision and Ophthalmology annual meeting 2008, Fort Lauderdale, FL, USA.

\begin{abstract}
Purpose To determine whether there were differences in the structure-function relationship between early and advanced glaucoma, and study the association between thickness of discrete macular cell layers, the thickness of the retinal nerve fiber layer, and visual field sensitivity.

Methods In all, 71 eyes of 50 subjects (28 glaucoma patients and 22 normal control subjects) were included. Thickness of macular retinal nerve fiber layer (mRNFL), macular inner retinal layer (mIRL), and macular outer retinal layer (mORL) were measured from Stratus optical coherence tomography macular scans, using our previously published segmentation algorithm. Visual sensitivity loss was determined by mean deviation (MD) using Humphrey Visual Field Analyzer. The mean thickness for each layer from the normal control subjects, early, and advanced glaucoma groups was compared. In addition, a mixed model analysis was used to explore the relationship between structure-function, allowing for possible interaction with glaucoma stage. Results The mean mRNFL thickness in early and advanced glaucoma patients was significantly less than measurements in normal subjects $(P<0.01)$. The mean mIRL thickness in advanced glaucoma was significantly less than normal subjects $(P=0.04)$. The mean mORL thickness in early and advanced glaucoma was not statistically significant different from that of normal subjects $(P>0.8)$. There was no statistically significant difference in macular structurefunction relationship between the two glaucoma groups $(P>0.05)$. Mean $\mathrm{mIRL}$ thickness was significantly associated with MD $(P=0.04)$.
\end{abstract}

TS Vajaranant', RJ Anderson², R Zelkha', C Zhang'1, JT Wilensky', DP Edward ${ }^{3}$ and M Shahidi'

Conclusion There was no significant difference in macular structure-function relationship between early and advanced glaucoma groups. Combined data from both glaucoma groups indicated that mIRL thickness was associated with visual sensitivity loss.

Eye (2011) 25, 612-618; doi:10.1038/eye.2011.17; published online 25 February 2011

Keywords: glaucoma; macular thickness; retinal nerve fiber; optical coherence tomography

Introduction

The clinical management of glaucoma relies on accurately identifying and monitoring structural and functional damage before the development of irreversible vision loss. Objective assessment of structural parameters such as peripapillary retinal nerve fiber layer (pRNFL) thickness measurement as well as subjective macular function assessment using psychophysical testing such as short wave length and frequency doubling perimetry has been used for the detection of early glaucoma. ${ }^{1-8}$ Because psychophysical testing is subjective, it can be highly variable and depends largely on the subject's cooperation. Therefore, objective structural testing, pRNFL thickness measurement has been used, as an adjunct to visual field testing for the detection of early glaucoma. ${ }^{1-8}$ However, the measurement of pRNFL thickness alone is of limited use as a screening tool for detection of glaucoma because of the large thickness variation in normal subjects. ${ }^{9,10}$ Furthermore, assessment of pRNFL thickness may not be 
useful for monitoring disease progression in advanced glaucoma. ${ }^{11}$

Recent technological advances in analysis of optical coherence tomography (OCT) macular images have shown a potential for identifying glaucomatous thickness changes in specific retinal layers. ${ }^{12-14}$ In this study, we applied an image segmentation algorithm developed by us to verify the extent of thinning of inner retinal cell layers due to glaucoma, compare macular structure-function relationship between early and advanced glaucoma, and determine the significance of association between thickness of discrete macular cell layers, the thickness of the retinal nerve fiber layer, and visual sensitivity loss. ${ }^{15}$

\section{Materials and methods}

This cross-sectional study was conducted at the University of Illinois at Chicago, Chicago, IL, USA. Each participant signed an informed consent document, which was approved by an Institutional Review Board at the University of Illinois.

The diagnosis of glaucoma was based on presence of characteristic glaucomatous optic neuropathy with loss of neuroretinal rim, with or without corresponding visual field defect. All glaucoma subjects underwent comprehensive ophthalmic evaluation and Humphrey Field testing (Carl Zeiss Meditec, Dublin, CA, USA). The visual field sensitivity was measured by Humphrey Visual Field Analyzer (Carl Zeiss Meditec) using the program 24-2, Swedish interactive threshold algorithm standard.

The degree of visual function damage was determined based on one reproducible visual field. Visual field defect was defined as abnormal glaucoma hemifield tests and/or the presence of three or more significant non-edge points including a point with at least one at the $P<0.5 \%$ on pattern deviation plots. ${ }^{16,17}$ The severity was determined by mean deviation (MD). For an eye to be included in the early glaucoma group the MD was greater than $-6 \mathrm{~dB}$ and for an eye to be included in the advanced glaucoma group, MD was equal to or less than $-6 \mathrm{~dB} .{ }^{16,17}$ Seven eyes with glaucomatous optic nerve damage without visual field defects were included in the early glaucoma group. Exclusion criteria included visual acuity worse than 20/40, unreliable visual field (defined as $>25 \%$ fixation losses, or $33 \%$ false negative and false positive), coexistent retinal disease, or optic nerve structural abnormalities other than cupping, moderate to severe media opacities, and inability to obtain optimal OCT images. The normal control subjects were selected from amongst employees of the UIC Eye Center and their relatives and associates. None of these subjects had ocular hypertension, retinal, or optic nerve abnormalities.

In all, 71 eyes of 50 subjects, 28 glaucoma patients (66 \pm 11 years), and 22 normally sighted control subjects
(58 \pm 11 years), were recruited for the study. Glaucomatous eyes were classified as early $(N=24)$ or advanced $(N=25)$. In the control group, there were 20 White, 1 African American, and 1 Hispanic subjects, and there were 14 females and 9 males. In the glaucoma group, there were 8 White, 16 African American, 3 Hispanic, and 1 Asian subjects, and there were 14 females and 14 males. In 21 glaucoma patients, both eyes were eligible for the study. The control subjects were 8 years younger than the glaucoma patients $(P=0.02)$.

\section{Image acquisition and analysis}

OCT imaging was performed using a Stratus OCT (Carl Zeiss Meditec). Six radial macular OCT scans, each $6 \mathrm{~mm}$ in length, were acquired. Each radial OCT scan was 1024 pixels $(2 \mathrm{~mm})$ in depth and 512 pixels $(6 \mathrm{~mm})$ in length. The depth and lateral resolutions were 10 and $20 \mu \mathrm{m}$, respectively. The raw grayscale OCT images were exported for analysis. All OCT images had signal strengths $\geqslant 5$ and displayed clear boundaries stratifying retinal layers. Our previously developed software program was used to segment the OCT image by the following steps: (1) alignment of A-lines; (2) gray-level mapping; (3) directional filtering; (4) edge detection; and (5) model-based decision making. ${ }^{15}$ The software allowed thickness measurement of three retinal layers, whereas the instrument's software only allows measurement of total retinal thickness. On each OCT image, three boundaries were detected automatically by the segmentation algorithm. These boundaries were (1) inner limiting membrane; (2) boundary between RNFL and inner plexiform layer and ganglion cell layer (IPL + GCL); and (3) inner and outer photoreceptor interface. The algorithm measured thickness of the following three retinal layers: RNFL, inner retina layer $(\mathrm{IRL}=\mathrm{IPL}+\mathrm{GCL}+\mathrm{INL}+\mathrm{OPL})$, and outer retina layer $(\mathrm{ORL}=$ outer nuclear layer and photoreceptor inner segments). These three retinal layers are marked on a sample OCT scan shown in Figure 1. Thickness measurements derived from six radial scans were combined to generate a thickness map for RNFL, IRL, and ORL in each eye. The main outcome measures were means for mRNFL, macular IRL (mIRL), and macular ORL (mORL) thickness. The repeatability of mRNFL, mIRL, and mORL thickness measurements were previously established to be 3,15 , and $6 \mu \mathrm{m}$, respectively. ${ }^{15}$

\section{Statistical analysis}

The mean thickness for each retinal layer measured by OCT in the three groups, normal subjects, early, and advanced glaucoma, was compared using 
Tukey-adjusted comparisons. The relationship between structural measures (mean thickness for each macular layer) and functional measures (MD) was determined for early and advanced glaucoma using mixed model analysis, which adjusted for the correlation between measurements obtained from both eyes of the same individual. The interaction test was used to determine the difference of the structure-function relationship

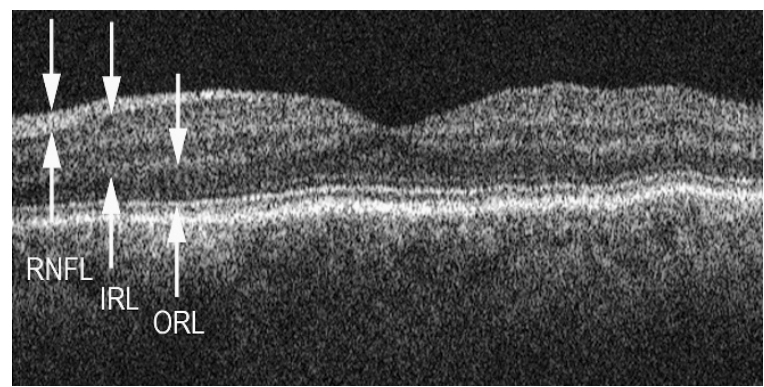

Figure 1 A typical example of a horizontal OCT radial scan from a left eye. The left and right sides of the image correspond to nasal and temporal retina, respectively. The image segmentation algorithm automatically detected retinal cell layer boundaries and measured thickness of retinal nerve fiber layer (RNFL), inner retina layer (IRL), and outer retina layer (ORL). These three retinal layers are marked by arrows on the image. between the two glaucoma stages. When the interaction test was not significant, it was removed from the model, allowing the determination of the association between structural measures and functional measures across both groups to be analyzed. All statistical analyses were performed with SAS version 9.1 (SAS Institute, Cary, NC, USA). A $P$-value $<0.05$ was considered to be statistically significant.

\section{Results}

Compiled mRNFL, mIRL, and mORL thickness maps generated by averaging measurements obtained in normal subjects, early, and advanced glaucoma patients are shown in Figure 2. The mRNFL thickness was highest near the optic nerve head and a considerable thinning was observed in early and advanced glaucoma group as compared with normal subjects. The mIRL thickness showed increased thickness in the parafoveal ring and was thinner in early and advanced glaucoma as compared with normal subjects. The mORL thickness was not significantly different in glaucoma groups as compared with normal subjects.

The average MD was $-1.5 \pm 2.1 \mathrm{~dB}$ for the early glaucoma group $(N=24)$, and $-12.9 \pm 5.9 \mathrm{~dB}$ for the
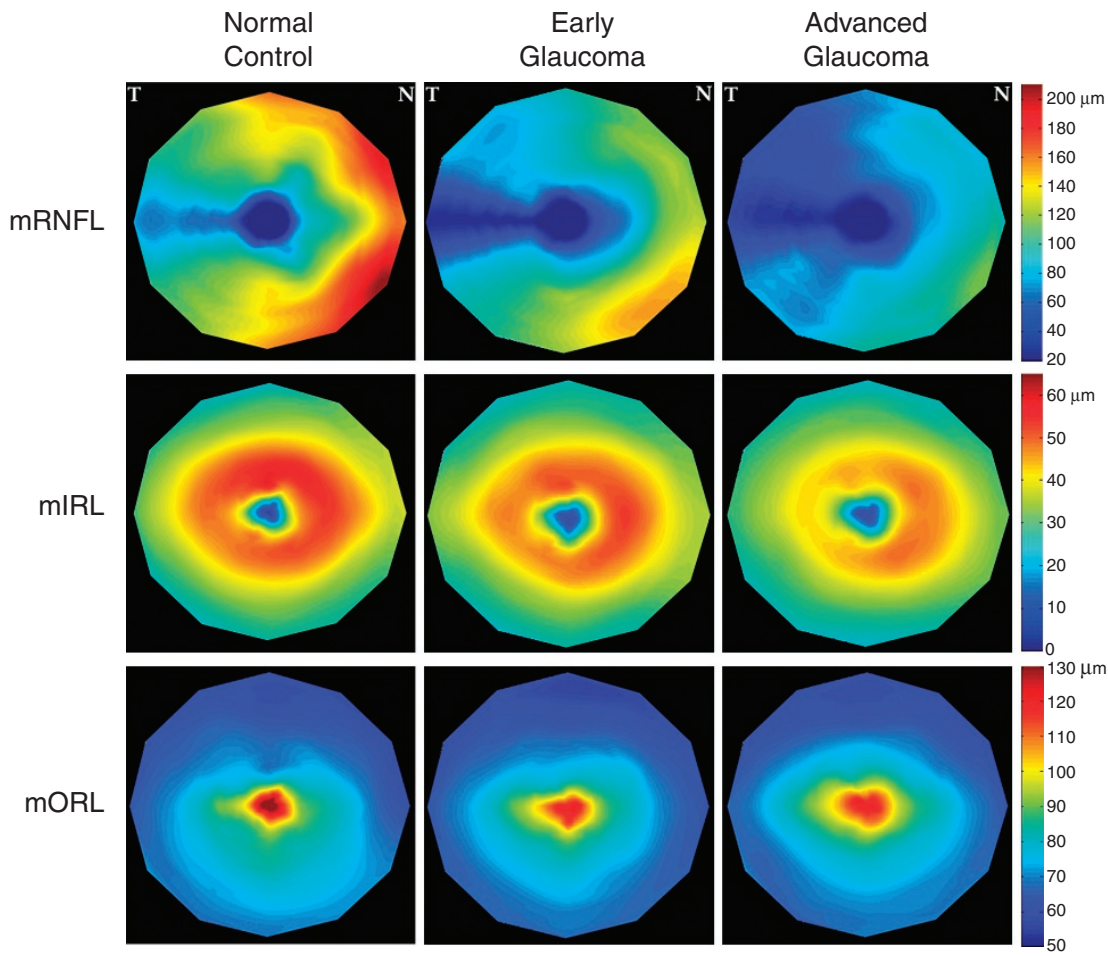

Figure 2 Compiled thickness maps generated from data in normal, early, and advanced glaucoma subjects. Combined maps correspond to a right eye with nasal (N) and temporal (T) retina marked on the right and left upper corners, respectively. Thickness maps of macular retinal nerve fiber layer (mRNFL), macular inner retina layer (mIRL), and macular outer retina layer (mORL) are shown in pseudocolor. Color bar represents thickness values in microns. 
Table 1 Comparison of mean thickness for macular cell layers among normally sighted control, early, and advanced glaucoma groups

\begin{tabular}{|c|c|c|c|c|c|c|c|}
\hline \multirow[t]{2}{*}{ Retinal cell layer } & \multicolumn{3}{|c|}{ Thickness (mean \pm SEM in $\mu m$ ) } & \multicolumn{3}{|c|}{ Tukey-adjusted comparison P-values } & \multirow{2}{*}{$\begin{array}{l}\text { P-values } \\
\text { Among all } \\
\text { groups }\end{array}$} \\
\hline & $\begin{array}{l}\text { Control } \\
(\mathrm{N}=22)\end{array}$ & $\begin{array}{c}\text { Early } \\
(\mathrm{N}=24)\end{array}$ & $\begin{array}{c}\text { Advanced } \\
(\mathrm{N}=25)\end{array}$ & $\begin{array}{l}\text { Control } \\
\text { vs early }\end{array}$ & $\begin{array}{l}\text { Control vs } \\
\text { advanced }\end{array}$ & $\begin{array}{l}\text { Early vs } \\
\text { advanced }\end{array}$ & \\
\hline mRNFL & $35 \pm 2$ & $24 \pm 2$ & $16 \pm 2$ & $0.0009^{*}$ & $<0.0001^{*}$ & $0.003^{*}$ & $<0.0001^{*}$ \\
\hline mIRL & $135 \pm 3$ & $128 \pm 3$ & $125 \pm 3$ & 0.22 & $0.04^{*}$ & 0.45 & $0.05^{*}$ \\
\hline mORL & $73 \pm 2$ & $72 \pm 2$ & $73 \pm 2$ & 0.85 & 0.99 & 0.80 & 0.78 \\
\hline
\end{tabular}

Abbreviations: mIRL, macular inner retinal layer; mORL, macular outer retinal layer; mRNFL, macular retinal nerve fiber layer.

Asterisks $(*)$ indicate statistical significance $(P<0.05)$.

advanced glaucoma group $(N=25)$. The average cup to disc ratio was $0.78 \pm 0.11$ for the early glaucoma group $(N=24)$, and $0.86 \pm 0.08$ for the advanced glaucoma group $(N=25)$. Table 1 summarizes the comparison of mean thickness and SEM for macular cell layers for the three groups. The mean mRNFL thickness in the advanced glaucoma $(16 \pm 2 \mu \mathrm{m} ; N=25)$ was significantly lower than thickness in early glaucoma $(24 \pm 2 \mu \mathrm{m}$; $N=24 ; P=0.003)$. The mean mRNFL thickness in early and advanced glaucomatous eyes was significantly lower as compared with thickness in normal eyes ( $35 \pm 2 \mu \mathrm{m}$; $N=22 ; P<0.001$ ).

The mean mIRL thickness in advanced glaucomatous eyes $(125 \pm 3 \mu \mathrm{m})$ was significantly lower as compared to thickness in normal eyes $(P=0.04)$. However, there was no statistically significant difference in the mIRL thickness between early glaucomatous $(128 \pm 3 \mu \mathrm{m})$ and normal eyes $(135 \pm 3 \mu \mathrm{m} ; P=0.22)$. In addition, there was no statistically significant difference in the mORL thickness between advanced ( $73 \pm 2 \mu \mathrm{m})$ or early glaucomatous $(72 \pm 2 \mu \mathrm{m})$ and normal eyes $(73 \pm 2 \mu \mathrm{m}$; $P>0.8$.

The interaction test showed that the structure-function relationship was not significantly different between early and advanced glaucoma patients $(P=0.40$ for mRNFL vs MD; $P=0.39$ for mIRL vs MD; and $P=0.35$ for mORL vs MD) Given that result, the association between the structure-function measures across both groups was determined. Of all macular cells layers, regression analysis showed that mean mIRL thickness was significantly associated with MD ( $P=0.04$; Table 2$)$. Specifically, the regression line slope of 0.12 estimates that decrease in the mIRL thickness by $10 \mu \mathrm{m}$ would yield an approximate $1.2 \mathrm{~dB}$ decrease in functional measure (MD). Figure 3 shows scatter plots between structure-function for each macular layer.

\section{Discussion}

In this study, we applied our previously developed image segmentation algorithm to investigate the
Table 2 The association between thickness of macular cell layers and visual field sensitivity loss

\begin{tabular}{lcc}
\hline $\begin{array}{l}\text { Macular cell layer thickness vs } \\
\text { visual field sensitivity loss }\end{array}$ & Slope & P-values \\
\hline mRNFL vs mean deviation & 0.11 & 0.13 \\
mIRL vs mean deviation & 0.12 & $0.04^{*}$ \\
mORL vs mean deviation & -0.05 & 0.58 \\
\hline
\end{tabular}

Abbreviations: $\mathrm{mIRL}$, macular inner retinal layer; mORL, macular outer retinal layer; mRNFL, macular retinal nerve fiber layer. Asterisks $\left(^{*}\right)$ indicate statistical significance $(P<0.05)$.

presence of thinning of $\mathrm{mRNFL}$ and $\mathrm{mIRL}(\mathrm{GCL}+\mathrm{IPL})$ in early and advanced glaucoma. From our cohort, thinning of mRNFL was present in both early and advanced glaucoma, whereas the mIRL thickness was significantly reduced only in advanced glaucoma. Furthermore, we found no statistically significant difference in the structure-function relationship between early and advanced glaucoma. Of all macular cell layers, the mIRL thickness was significantly associated with glaucomatous visual field sensitivity loss.

We found that measurement of mRNFL thickness had the ability to distinguish early (MD, $-1.5 \mathrm{~dB}$ ) and advanced (MD, $-12.9 \mathrm{~dB}$ ) glaucomatous changes, consistent with previous reports. ${ }^{13,14}$ However, mIRL $(\mathrm{GCL}+\mathrm{IPL}+\mathrm{INL}+\mathrm{OPL})$ thickness was similar in early glaucoma and normal subjects. This finding differed from the results reported by Tan et al who found a statistically significant difference in GCL + IPL thickness between glaucoma suspect/pre-perimetric glaucoma $(\mathrm{MD}=0.13 \mathrm{~dB})$ and control groups. In contrast to this study, Tan $e$ al $^{14}$ reported an area-weighted thickness average excluding the fovea $(1.5 \mathrm{~mm})$, which may have contributed to the difference in our findings. However, our observation is supported by findings of a previous experimental glaucoma study that demonstrated a reduction in axon thickness before shrinkage of the ganglion cell soma suggesting mRNFL thinning would be more evident in the early glaucoma. ${ }^{5,18}$ In advanced 


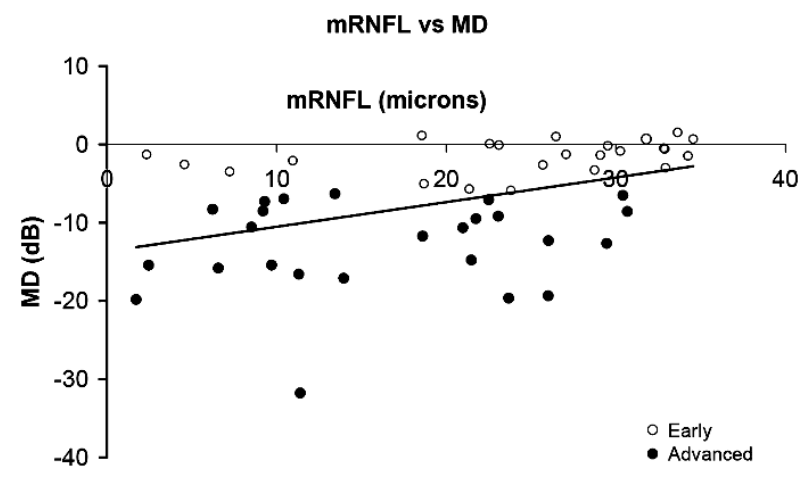

mIRL vs MD

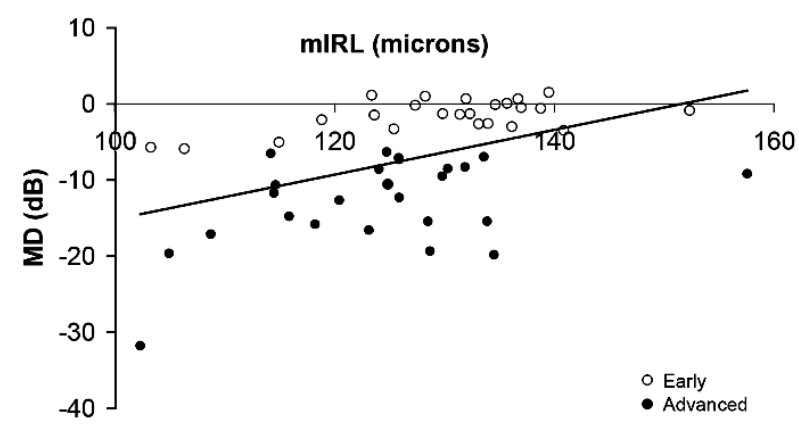

MORL vs MD

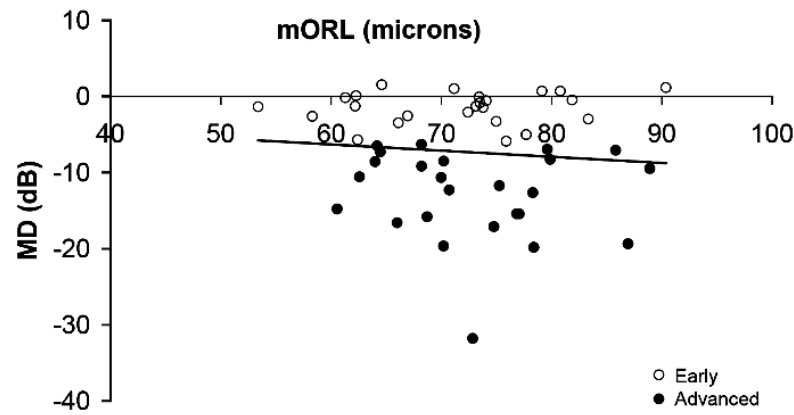

Figure 3 Relationship between thickness of macular cell layers and visual sensitivity loss.

glaucoma with the presence of greater retinal ganglion cell loss, thinning of the mIRL is expected to become more prominent, consistent with our findings. When compared with mRNFL thickness, the mIRL thickness was reduced to a lesser degree in glaucoma patients. This may be because the ganglion cell soma is less affected in early glaucoma and/or there may be an increase in nonneuronal tissue after ganglion cells degenerate.

In this study, we found a lack of significant correlation between mRNFL thickness and visual sensitivity loss. However, Leung $e t a l^{5}$ reported highly significant but moderate correlation $(r=0.39 ; P<0.001)$ between the $\mathrm{mRNFL}$ and MD. It is difficult to directly compare our results with Leung's report, as it was not clear whether they combined the data from normal, glaucoma suspect, and glaucoma groups. Furthermore, the glaucoma patients in our study had a greater loss of visual sensitivity and reduction in mRNFL thickness, which may have caused our data to be more susceptible to the 'floor' effect. Specifically, the presence of extensive damage with very thin RNFL tends to reduce signal-to-noise ratio.

In addition, the lack of significant difference in structure-function relationship between the early and advanced groups may be attributed to the fact that both groups had significant visual functional loss and optic disc morphological abnormalities, reflecting a more advanced stage on the glaucoma continuum. Nevertheless, our analysis showed that the mIRL thickness, not mRNFL was significantly associated with glaucomatous visual sensitivity loss. This finding suggests that mIRL thickness measurement might be useful for monitoring disease progression, as an adjunct to the visual field testing, after the diagnosis of glaucoma is established. Our analysis estimates that decrease in the mIRL thickness by $10 \mu \mathrm{m}$ would yield an approximate $1.2 \mathrm{~dB}$ decrease in functional measure (MD).

Our study had some limitations. First, our normal control group was younger than glaucoma groups. However, we found on average 30 and 52\% thinning of the mRNFL and 3 and $8 \%$ thinning of the mIRL in early and advanced glaucoma, respectively. The magnitude of these abnormalities exceeds what would be expected from age-related changes alone $(0.2-0.27 \%$ per year for RNFL and $0.5-0.6 \%$ per year for GCL).${ }^{19}$ Similarly, it is not likely that our results were affected by the racial differences between our control and glaucoma groups (predominately Caucasian in the control group and African American in the glaucoma group). Currently normative data on racial differences in the thickness for each retinal layer is not available. Previous studies reported a difference of only $2-5 \%$ in total macular thickness within the inner 3-mm sector between African Americans and Caucasians and no significant difference in the 6-mm outer sectors. ${ }^{20,21}$ Second, refraction and axial length might affect the OCT measurements; however, these data were not included, as they were not available for all subjects. ${ }^{22}$ Third, visual function as measured by Humphrey visual field 24-2 covers some area outside tested retinal thickness mapping $(6 \times 6 \mathrm{~mm})$. Given the characteristic pattern of RNFL in the macula, as retinal nerve fibers within the central macula extend over temporal retina (nasal visual field), it is estimated that the central macular scans would correspond to $\sim 60 \%$ of an area tested by Humphrey 24-2. The 24-2 protocol was selected for the study, as it is a gold standard for visual field testing and it includes the nasal 
visual field, which is commonly affected in early glaucoma. In addition, the 24-2 protocol is not only fast and reliable, but also provides MD, representing the overall stage of glaucoma. Lastly, as averaged values of thickness and visual field loss were used for analysis, mild and/or local abnormalities in early glaucoma were likely disregarded. Nonetheless, despite limitations, the presented protocol and analysis demonstrate that averaged mRNFL thickness, not averaged mIRL thickness could detect early changes in glaucoma and averaged mIRL thickness was significantly correlated with overall visual function (MD) after glaucomatous changes were established.

In conclusion, our findings suggest that mean mRNFL thickness assessment may be appropriate for early detection of glaucoma, whereas mean mIRL thickness may be better suited for monitoring disease progression after glaucoma diagnosis is established. Overall, thickness measurement of macular retinal layers by OCT has a potential to complement both peripapillary structural and macular functional measures and help improve the clinical management of glaucoma patients. Future studies using spectral-domain OCT technology with eye-tracking capability will allow local comparative assessment of structural-function relationship in glaucoma.

\section{Summary}

What was known before

- Structural changes in glaucoma are associated with retinal nerve fibre layer loss and retinal ganglion cell death.

- Recent technological advances in analysis of optical coherence tomography macular images have shown a potential for identifying glaucomatous thickness changes in specific retinal layers.

- A comparison of thickness changes in macular cell layers and their relationship to visual functional measures in early and advanced glaucoma has not been performed.

What this study adds

- In this study, we applied our segmentation algorithm to establish the presence of thinning of inner retinal cell layers due to glaucoma, compare macular structurefunction relationship between early and advanced glaucoma, and determine the significance of association between thickness of each macular cell layer and visual sensitivity loss.

- We found that there was no significant difference in macular structure-function relationship between early and advanced glaucoma groups. Combined data from both glaucoma groups indicated that mIRL thickness was associated with visual sensitivity loss.

\section{Conflict of interest}

The authors declare no conflict of interest.

\section{Acknowledgements}

This study is supported by the American Glaucoma Society (Young Clinician-Scientist Award, TSV), Department of Veterans Affairs, Washington, DC, NIH grants EY14275 (MS), and EY01792 (UIC core grant), Bethesda, Maryland; Senior Scientific Investigator Award (MS), and an unrestricted departmental grant from Research to Prevent Blindness Inc., New York, NY.

\section{References}

1 Fogagnolo P, Rossetti L, Ranno S, Ferreras A, Orzalesi N. Short-wavelength automated perimetry and frequencydoubling technology perimetry in glaucoma. Prog Brain Res 2008; 173: 101-124.

2 Guedes V, Schuman JS, Hertzmark E, Wollstein G, Correnti A, Mancini R et al. Optical coherence tomography measurement of macular and nerve fiber layer thickness in normal and glaucomatous human eyes. Ophthalmology 2003; 110(1): 177-189.

3 Kanamori A, Nakamura M, Escano MF, Seya R, Maeda H, Negi A. Evaluation of the glaucomatous damage on retinal nerve fiber layer thickness measured by optical coherence tomography. Am J Ophthalmol 2003; 135: 513-520.

4 Nouri-Mahdavi K, Hoffman D, Tannenbaum DP, Law SK, Caprioli J. Identifying early glaucoma with optical coherence tomography. Am J Ophthalmol 2004; 137: 228-235.

5 Leung CK, Chan WM, Yung WH, Ng AC, Woo J, Tsang MK et al. Comparison of macular and peripapillary measurements for the detection of glaucoma: an optical coherence tomography study. Ophthalmology 2005; 112(3): 391-400.

6 Budenz DL, Michael A, Chang RT, McSoley J, Katz J. Sensitivity and specificity of the Stratus OCT for perimetric glaucoma. Ophthalmology 2005; 112: 3-9.

7 Ojima T, Tanabe T, Hangai M, Yu S, Morishita S, Yoshimura N. Measurement of retinal nerve fiber layer thickness and macular volume for glaucoma detection using optical coherence tomography. Jpn J Ophthalmol 2007; 51(3): 197-203.

8 Parikh RS, Parikh S, Sekhar GC, Kumar RS, Prabakaran S, Babu JG et al. Diagnostic capability of optical coherence tomography (Stratus OCT 3 ) in early glaucoma. Ophthalmology 2007; 114(12): 2238-2243.

9 Sanchez-Galeana C, Bowd C, Blumenthal EZ, Gokhale PA, Zangwill LM, Weinreb RN. Using optical imaging summary data to detect glaucoma. Ophthalmology 2001; 108: 1812-1818.

10 Greaney MJ, Hoffman DC, Garway-Heath DF, Nakla M, Coleman AL, Caprioli J. Comparison of optic nerve imaging methods to distinguish normal eyes from those with glaucoma. Invest Ophthalmol Vis Sci 2002; 43: 140-145.

11 Blumenthal EZ, Horani A, Sasikumar R, Garudadri C, Udaykumar A, Thomas R. Correlating structure with function in end-stage glaucoma. Ophthalmic Surg Lasers Imaging 2006; 37(3): 218-223.

12 Zeimer R, Asrani S, Zou S, Quigley H, Jampel H. Quantitative detection of glaucomatous damage at the posterior pole by retinal thickness mapping. A pilot study. Ophthalmology 1998; 105(2): 224-231. 
13 Ishikawa H, Stein DM, Wollstein G, Beaton S, Fujimoto JG, Schuman JS. Macular segmentation with optical coherence tomography. Invest Ophthalmol Vis Sci 2005; 46(6): 2012-2017.

14 Tan O, Li G, Lu AT, Varma R, Huang D. Advanced Imaging for Glaucoma Study Group. Mapping of macular substructures with optical coherence tomography for glaucoma diagnosis. Ophthalmology 2008; 115(6): 949-956.

15 Bagci AM, Shahidi M, Ansari R, Blair M, Blair NP, Zelkha R. Thickness profiles of retinal layers by optical coherence tomography image segmentation. Am J Ophthalmol 2008; 146(5): 679-687.

16 Hodapp E, Parrish RK, Anderson DR. Clinical Decisions in Glaucoma. Mosby-Year Book: St Louis, 1993; 3-63.

17 Anderson DR, Patella VM. Automated static perimetry. 2nd edn, Mosby: St Louis, 1999; 121-126.

18 Weber AJ, Kaufman PL, Hubbard WC. Morphology of single ganglion cells in the glaucomatous primate retina. Invest Ophthalmol Vis Sci 1998; 39(12): 2304-2320.
19 Harwerth RS, Wheat JL, Rangaswamy NV. Age-related losses of retinal ganglion cells and axons. Invest Ophthalmol Vis Sci 2008; 49(10): 4437-4443.

20 Girkin CA, Sample PA, Lieberman JM, Jain S, Bowd C, Becerra LM et al. African Descent and Glaucoma Evaluation Study (ADAGES): II. Ancestry differences in optic disc, retinal nerve fiber layer, and macular structure in healthy subjects. Arch Ophthalmol 2010; 128(5): 541-550.

21 Kelty PJ, Payne JF, Trivedi RH, Kelty J, Bowie EM, Burger BM. Macular thickness assessment in healthy eyes based on ethnicity using Stratus OCT optical coherence tomography. Invest Ophthalmol Vis Sci 2008; 49(6): 2668-2672.

22 Wu PC, Chen YJ, Chen CH, Chen YH, Shin SJ, Yang HJ et al. Assessment of macular retinal thickness and volume in normal eyes and highly myopic eyes with third-generation optical coherence tomography. Eye (Lond) 2008; 22(4): 551-555. 